\title{
First stellar spectroscopy in Leo P
}

\author{
C. J. Evans ${ }^{1}$, N. Castro ${ }^{2,3}$, O. A. Gonzalez ${ }^{1}$, M. Garcia ${ }^{4}$, N. Bastian ${ }^{5}$, M.-R. L. Cioni ${ }^{3}$, J. S. Clark ${ }^{6}$, B. Davies ${ }^{5}$, \\ A. M. N. Ferguson ${ }^{7}$, S. Kamann ${ }^{5}$, D. J. Lennon ${ }^{8,9}$, L. R. Patrick ${ }^{9,10}$, J. S. Vink ${ }^{11}$, and D. R. Weisz ${ }^{12}$ \\ ${ }^{1}$ UK Astronomy Technology Centre, Royal Observatory, Blackford Hill, Edinburgh EH9 3HJ, UK \\ e-mail: chris.evans@stfc.ac.uk \\ 2 Department of Astronomy, University of Michigan, 1085 S. University Avenue, Ann Arbor, MI 48109-1107, USA \\ 3 Leibniz-Institut für Astrophysik Potsdam, An der Sternwarte 16, 14482 Potsdam, Germany \\ ${ }^{4}$ Centro de Astrobiología, CSIC-INTA, Ctra. Torrejón a Ajalvir km.4, 28850 Torrejón de Ardoz, Madrid, Spain \\ 5 Astrophysics Research Institute, Liverpool John Moores University, Liverpool L3 5RF, UK \\ 6 Department of Physics and Astronomy, The Open University, Walton Hall, Milton Keynes MK7 6AA, UK \\ 7 Institute for Astronomy, University of Edinburgh, Blackford Hill, Edinburgh EH9 3HJ, UK \\ 8 ESA, European Space Astronomy Centre, Apdo. de Correos 78, 28691 Villanueva de la Cañada, Madrid, Spain \\ 9 Instituto de Astrofísica de Canarias, 38205 La Laguna, Tenerife, Spain \\ 10 Universidad de La Laguna, Dpto. Astrofísica, 38206 La Laguna, Tenerife, Spain \\ 11 Armagh Observatory and Planetarium, College Hill, Armagh BT61 9DG, UK \\ 12 Department of Astronomy, University of California Berkeley, Berkeley, CA 94720, USA
}

Received 27 August 2018 / Accepted 21 December 2018

\begin{abstract}
We present the first stellar spectroscopy in the low-luminosity $\left(M_{V} \sim-9.3 \mathrm{mag}\right)$, dwarf galaxy Leo P. Its significantly low oxygen abundance $(3 \%$ solar) and relative proximity $(\sim 1.6 \mathrm{Mpc})$ make it a unique galaxy in which to investigate the properties of massive stars with near-primordial compositions akin to those in the early Universe. From our VLT-MUSE spectroscopy we find the first direct evidence for an O-type star in the prominent $\mathrm{H}$ II region, providing an important test case to investigate the potential environmental dependence of the upper end of the initial mass function in the dwarf galaxy regime. We classify 14 further sources as massive stars (and 17 more as candidate massive stars), most likely B-type objects. From comparisons with published evolutionary models we argue that the absolute visual magnitudes of massive stars in very metal-poor systems such as Leo P and IZw 18 may be fainter by $\sim 0.5$ mag compared to Galactic stars. We also present spectroscopy of two carbon stars identified previously as candidate asymptotic-giantbranch stars. Two of three further candidate asymptotic-giant-branch stars display Ca II absorption, confirming them as cool, evolved stars; we also recover Ca II absorption in the stacked data of the next brightest 16 stars in the upper red giant branch. These discoveries will provide targets for future observations to investigate the physical properties of these objects and to calibrate evolutionary models of luminous stars at such low metallicity. The MUSE data also reveal two $100 \mathrm{pc}$-scale ring structures in $\mathrm{H} \alpha$ emission, with the $\mathrm{H}$ II region located on the northern edge of the southern ring. Lastly, we report serendipitous observations of 20 galaxies, with redshifts ranging from $z=0.39$, to a close pair of star-forming galaxies at $z=2.5$.
\end{abstract}

Key words. stars: early-type - stars: AGB and post-AGB - galaxies: individual: Leo P

\section{Introduction}

Leo $\mathrm{P}$ is a relatively nearby, dwarf irregular galaxy. Its discovery and first studies of its physical properties were reported in a series of five papers by Giovanelli et al. (2013), Rhode et al. (2013), Skillman et al. (2013), McQuinn et al. (2013), and Bernstein-Cooper et al. (2014). Initially discovered from $\mathrm{HI}$ observations from the Arecibo Legacy Fast ALFA Survey (ALFALFA), further radio observations revealed its spatial extent and velocity structure (Giovanelli et al. 2013). Optical imaging from the $3.5 \mathrm{~m}$ WIYN telescope gave a first view of its stellar population, with evidence of ongoing star formation in a luminous H II region (Rhode et al. 2013). Spectroscopy of the $\mathrm{H}$ II region from the $4 \mathrm{~m}$ Mayall Telescope and the Large Binocular Telescope (LBT) was used to estimate an oxygen abundance, via analysis of the $[\mathrm{O} \mathrm{III}] \lambda 4363$ line, of $[\mathrm{O} / \mathrm{H}]=7.17 \pm 0.04$ (just $3 \%$ of solar, Skillman et al. 2013). We lack an estimate of iron abundance at present, but even if Leo $\mathrm{P}$ were found to have a sub-solar $\alpha / \mathrm{Fe}$ ratio (as found for massive stars in other metalpoor dwarf irregulars, e.g. Venn et al. 2003; Tautvaišienè et al.
2007; Garcia et al. 2014; Hosek et al. 2014), such a low oxygen abundance suggests it is one of the most metal-poor star-forming galaxies known ${ }^{1}$.

Follow-up imaging with the LBT was used by McQuinn et al. (2013) to estimate a distance of $1.72_{-0.40}^{+0.14} \mathrm{Mpc}$, from the location of the tip of the red giant branch (TRGB) in the colour-magnitude diagram (CMD), with an improved measurement of $1.62 \pm 0.15 \mathrm{Mpc}$ from the luminosity of the horizontal branch and light curves of candidate RR Lyrae stars (McQuinn et al. 2015). The system is thought to have a stellar mass of $5.7 \times 10^{5} M_{\odot}$ (McQuinn et al. 2013), with a neutral-hydrogen mass of $9.5 \times 10^{5} M_{\odot}$ and a dynamical mass in excess of $2.6 \times 10^{7} M_{\odot}$ (Bernstein-Cooper et al. 2014).

Following its discovery, Bellazzini et al. (2013) suggested Leo $\mathrm{P}$ is a member of the NGC 3109 association and is one of five "dwarfs walking in a row" (together with NGC 3109, Sextans A, Sextans B, and Antlia). They speculated this association

\footnotetext{
1 Hence the moniker of "Leo P" from Giovanelli et al. (2013), where "P" refers to its pristine nature.
} 


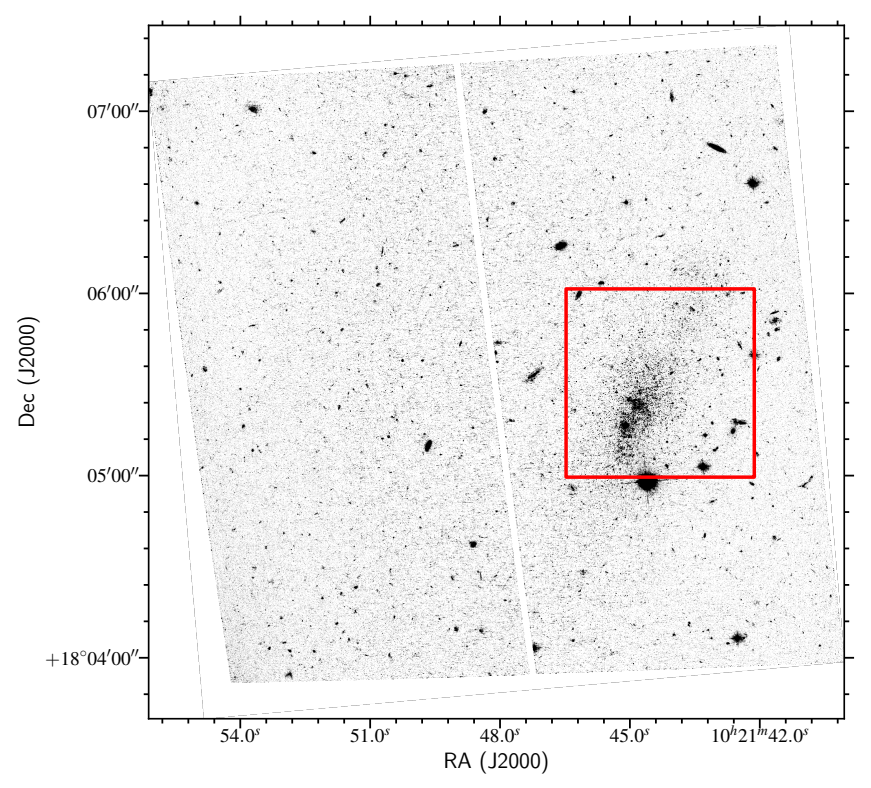

Fig. 1. Observed MUSE field (in red) overlaid on the F475W HSTACS image of the Leo P region (McQuinn et al. 2015). The MUSE data encompass most of the visible extent of this low-luminosity dwarf system.

could have originated from tidal interaction or, if arriving at the Local Group for the first time, that the structure could be a remnant of a cosmological filament.

In this context, Leo $\mathrm{P}$ is of interest as the potential site of the most metal-poor massive stars in the local Universe. The populations of high-mass stars in metal-poor galaxies are a prime contender for the intense ultraviolet radiation which reionised the intergalactic medium by $z \sim 6$ (e.g. Finkelstein et al. 2012; Stark 2016; Weisz \& Boylan-Kolchin 2017). However, while there are theoretical predictions for the properties of high-mass stars at these early epochs (including the putative "Population III" stars), we lack observational tests of this very metal-poor regime.

In the Local Group (and nearby), the range of lowmetallicity environments in which to study massive-star evolution is limited to the Magellanic Clouds (e.g. the VLT-FLAMES surveys, Evans et al. 2005, 2011) and other metal-poor galaxies such as IC 1613, WLM, NGC 3109 and Sextans A (e.g. Tramper et al. 2011, 2014; Garcia \& Herrero 2013; Camacho et al. 2016). To reach metallicities significantly below those in the Clouds for substantial stellar populations, we need to look much further afield to star-forming galaxies such as DDO $68(\sim 12.7 \mathrm{Mpc}$, Cannon et al. 2014; Sacchi et al. 2016) or I Zwicky 18 (18.2 $\pm 1.5 \mathrm{Mpc}$, Aloisi et al. 2007). The presence of young blue populations in Leo A and the Sag DIG (Weisz et al. 2014; Garcia 2018) and the discovery of Leo P, gives us a first chance to explore this regime. Tantalisingly, Leo $\mathrm{P}$ has a comparable oxygen abundance to both DDO 68 and IZw 18 (Skillman \& Kennicutt 1993; Pustilnik et al. 2005) but is signficantly closer, potentially providing a unique opportunity to study high-mass stars in a pristine environment similar to those in the earliest stages of the Universe.

The cool-star population of Leo $\mathrm{P}$ is of also interest. The main sites of dust production in the early Universe are thought to be supernovae (e.g. Michałowski et al. 2010) and stars on the asymptotic giant branch (AGB, e.g. Valiante et al. 2009; Zhukovska \& Henning 2013); luminous blue variables might also play a role (Guha Niyogi et al. 2014). Considerable effort has been undertaken to identify and characterise the AGB pop- ulations of local metal-poor galaxies to investigate this channel for dust production (e.g. Boyer et al. 2009, 2012, 2015, 2017; Sloan et al. 2009, 2012; Jones et al. 2018). Identification of AGB stars in Leo P would be a natural extension to such studies. Lee (2016) used single-epoch, near-IR imaging of Leo P to identify 22 candidate AGB stars, but used selection criteria from Sibbons et al. (2012) from their observations of NGC 6822 (where the cool stars are more metal-rich than Leo P, e.g. Patrick et al. 2015). Spectroscopic confirmation of their nature is a first step to planning longer-wavelength observations to investigate dust-production rates.

Here we present the first stellar spectroscopy in Leo P, obtained with the Multi-Unit Spectroscopic Explorer (MUSE; Bacon et al. 2010) on the Very Large Telescope (VLT) at Paranal. The observations and data reduction are detailed in Sect. 2, followed by discussion of the stellar spectra (and detections of background galaxies) in Sect. 3. In Sect. 4 we discuss the potential impact of the low metallicity of Leo P on the magnitudes of massive stars, and in Sect. 5 we briefly investigate the morphology and dynamics of the nebular emission. Concluding remarks are given in Sect. 6.

\section{Observations and data reduction}

We observed Leo $\mathrm{P}$ using the extended wide-field mode of MUSE, without the blue filter to extend the wavelength coverage down to $4650 \AA$ (to include He II $\lambda 4686$ if present in our targets). Eight observations, spanning 13 December 2015-8 March 2016, were executed from the service queue on UT4 of the VLT. Each comprised $2 \times 1505 \mathrm{~s}$ exposures (so a total integration of $6.7 \mathrm{~h}$ ), centred on a position of $\alpha=10^{\mathrm{h}} 21^{\mathrm{m}} 44.3, \delta=18^{\circ} 05^{\prime} 30^{\prime \prime} .5$ (J2000). As shown in Fig. 1, the observed MUSE field spans most of the visible extent of the galaxy. All of the observations were obtained in dark time (in terms of lunar illumination and angular distance), except for one that was on the boundary of grey and bright time.

The data were reduced using the MUSE pipeline (v1.6.1), with steps including: bias correction, wavelength calibration, reconstruction of datacubes from the individual spectra on the detectors, correction of the spectra to the heliocentric frame, sky subtraction, and merging of the individual exposures to form a combined datacube. Our reductions were largely indistinguishable to the "MUSE-DEEP" processing and stacking of the data undertaken as part of ESO's archiving activities, and the combined cube is available from the ESO Phase 3 archive ${ }^{2}$. The central parts of the cube are relatively crowded (cf. the typical seeing of the observations of $\left.\sim 00^{\prime \prime} 6\right)$. To extract our sources we used the PAMPELMUSE software (Kamann et al. 2013) that was developed to recover spectra from blended sources in MUSE data (see, e.g. Kamann et al. 2016).

For the input catalogue for the PAMPELMUSE extractions we used the Hubble Space Telescope (HST) imaging from McQuinn et al. (2015), who used the Advanced Camera for Surveys (ACS) to observe Leo $\mathrm{P}$ with the $F 475 \mathrm{~W}$ and $F 814 \mathrm{~W}$ filters (see Fig. 1). We created a source catalogue from the HST data with DOLPHOT, a version of HSTPHOT (Dolphin 2000, that has been updated with a specific module for ACS), and using the parameter file and photometric criteria from Williams et al. (2014). The angular resolution of these data is a factor of ten better than the MUSE observations, and many of the MUSE sources are resolved into multiple components.

\footnotetext{
2 http://archive.eso.org/wdb/wdb/adp/phase3_main/form
} 

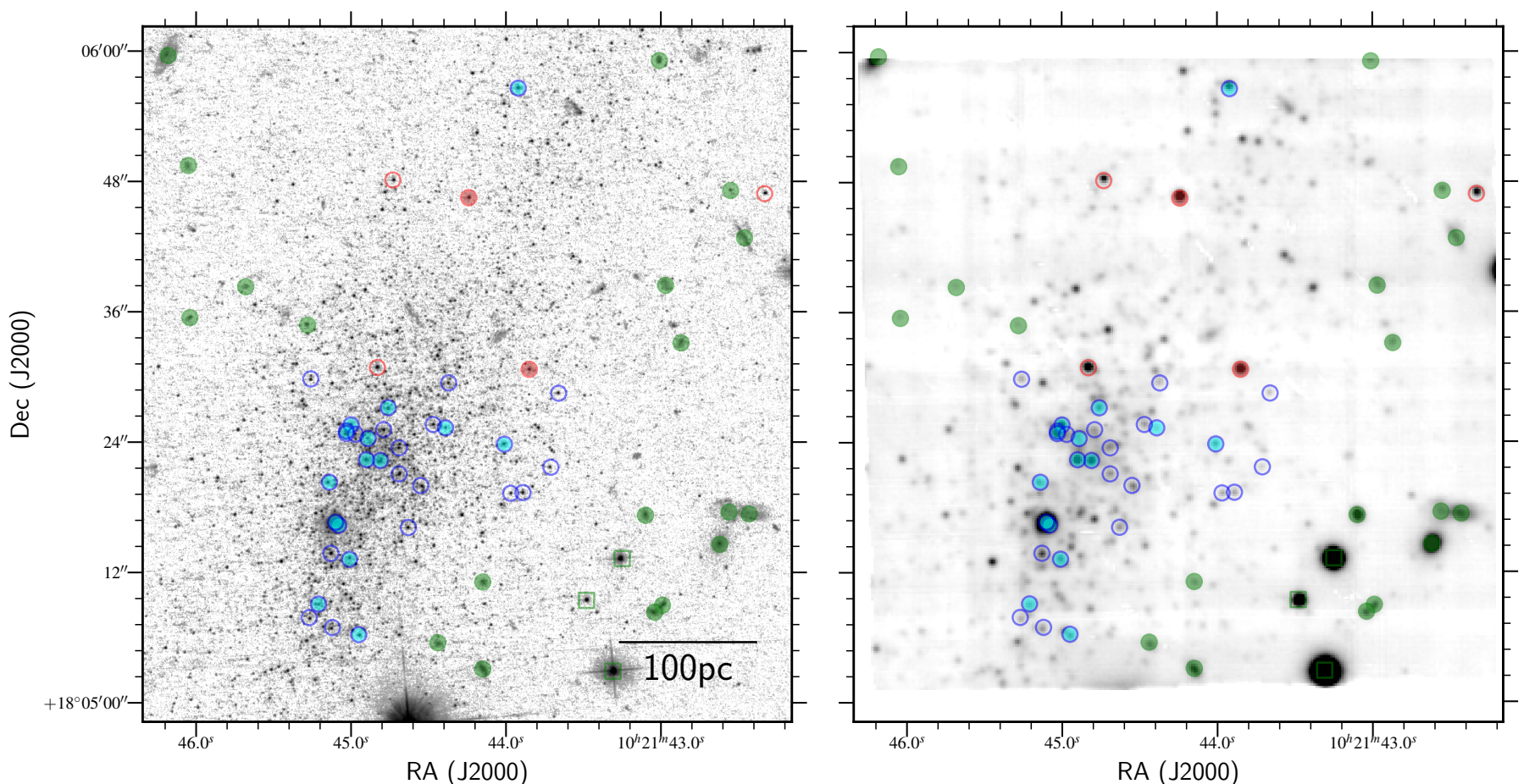

Fig. 2. Spatial distribution of classified spectra in Leo P. Left-hand panel: HST-ACS F475W image; right-hand panel: V-band image recovered from the MUSE cube. Objects are identified as follows: massive stars (filled cyan circles), candidate massive stars (open blue circles), carbon stars (filled red circles), additional candidate AGB stars from Lee (2016, open red circles), background galaxies (green circles), foreground stars (open green squares). The $100 \mathrm{pc}$ scalebar assumes the distance of $1.62 \mathrm{Mpc}$ from McQuinn et al. (2015).

PAMPELMUSE is designed to fit the point spread function (PSF) of each source throughout each wavelength slice of the combined MUSE datacube. In general, this requires a subset of bright, high signal-to-noise stars to define the PSF and coordinate transformations between the input catalogue and the MUSE observations prior to the extractions. Unfortunately, our Leo P field lacked sufficiently bright stars for adequate PSF modelling. This is in contrast to the recent analysis of the luminous stellar population of NGC 300 which, although more distant at $\sim 1.9 \mathrm{Mpc}$, has a much greater stellar density and a significant population of luminous stars (Roth et al. 2018).

We therefore used PAMPELMUSE to fit all resolved sources simultaneously, which entailed computing both the Gaussian PSF profile and the coordinate transformations at each layer. After PAMPELMUSE had selected all the stars that were suitable for fitting and extraction, we performed a first run using a binning of 50 pixels in the spectral direction. This allowed us to smooth the wavelength dependencies to the fitted parameters by using a simple polynomial fit before performing the final extraction at each wavelength for 341 sources from the HST catalogue. In the cases of well isolated stars the resulting spectra were comparable to simple aperture extractions from the cube. However, the PAMPELMUSE approach has the advantage over aperture extractions of not simply co-adding multiple components together in one spectrum (where objects are either blended or simply within the defined aperture).

The positions and photometry of the MUSE spectra that we were able to classify are given in Tables 1 and 2 . We also give the identifiers and photometry from the ground-based imaging from (MSB; McQuinn et al. 2013). Those data were obtained with a seeing of $0^{\prime \prime} .7$, so are a good match to the angular resolution of our MUSE cube for relatively isolated stars (or where additional components detected in the HST images are sufficiently faint not to contribute more than a few percent of the flux). Given the small field of the ACS images, the right ascension and declination of our sources are given in the astrometric frame of McQuinn et al. (2013), where the HST and MUSE data were transformed using matched stars from their catalogue.

\section{Spectral content}

Our motivation for the MUSE observations was a first spectroscopic census of Leo $P$, primarily to identify candidate massive stars for quantitative analysis with higher-quality, follow-up spectroscopy. The spatial locations of our classified spectra are shown in Fig. 2, with their positions in the HST CMD shown in Fig. 3. For reference, McQuinn et al. (2013) estimated the TRGB as $I=22.11 \mathrm{mag}$. As such, we expected to find a number of (modestly) massive stars in the MUSE spectroscopy in the "blue" plume at $(F 475 W-F 814 W) \sim 0.0$ mag, and luminous evolved red stars above the TRGB. We have found examples of both hot and cool objects, as well as background galaxies in the MUSE field, as outlined in the following sections.

\subsection{HIl region}

There is a prominent $\mathrm{H}$ II region in Leo $\mathrm{P}$, discussed by Skillman et al. (2013), and resolved into multiple sources and nebulosity by the HST images (McQuinn et al. 2015). Skillman et al. mentioned an absence of absorption features, but the MUSE spectrum of the bright, central star (LP 26) displays He II $\lambda \lambda 4686,5411$ absorption (see Fig. 4), arguing for the presence of an O-type star. McQuinn et al. (2015) estimated the absolute magnitude of this source as $M_{V}=-4.43$, equivalent to what we would expect for a mid to late O-type dwarf at Galactic metallicity (e.g. Walborn 1972; Martins et al. 2005). In the absence of published calibrations for the absolute magnitudes of O-type stars at low metallicities, we discuss this aspect further in Sect. 4. 


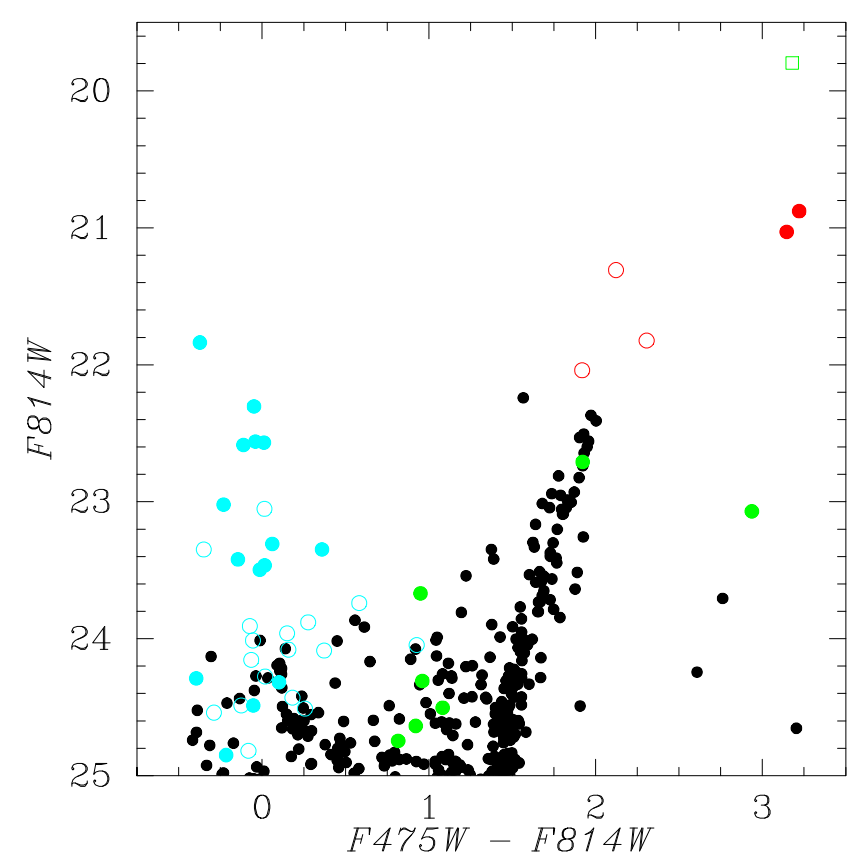

Fig. 3. Colour-magnitude diagram for the MUSE field using our photometry of the HST imaging from McQuinn et al. (2015). Symbols as follows: massive stars (closed cyan circles), candidate massive stars (open cyan circles), carbon stars (closed red circles), other sources previously classified as potential AGB stars (open red circles), background galaxies (green circles), foreground star (open green square).

\subsection{Early-type stars}

At the spectral resolution $(R \sim 2000$ at $5500 \AA)$ and signal-tonoise $(\mathrm{S} / \mathrm{N})$ of the MUSE data, we would not expect to be able to identify many prominent spectral lines in early-type stars (which is further exacerabated by the lack of spectral coverage shortwards of $\sim 4650 \AA$ ). Nonetheless, on the basis of $\mathrm{H} \beta$ absorption, combined with indications of stellar features at $\mathrm{H} \alpha$, we classify 14 sources as massive stars, with spectra of some of the brighter sources shown in Fig. 5 (with a $\mathrm{S} / \mathrm{N}$ ranging from 12 to 28 per pixel). We classified a further 17 spectra as possible massive stars, typically where the $\mathrm{H} \beta$ absorption is less secure, but still supported by an increasingly blue flux distribution.

Given this data quality, combined with the spectral range, sky residuals, and resolution of MUSE, it is not surprising that we do not see other strong features. That said, weak absorption consistent with arising from He I $\lambda \lambda 5876,6678$ is seen in both LP 16 and LP 18 (see Fig. 5), but we also see evidence for oversubtraction of the [O III] nebular lines (and similarly in the core of $\mathrm{H} \alpha$ ) suggesting these could be related to limitations of the background subtraction. The only other feature of note is Paschen $9 \lambda 9229$ absorption in the spectra of LP 21 and LP 23. From the steepening blue flux distributions of these spectra, combined with an absence of features that might be expected at cooler types, we expect that most of these are B-type objects (or potentially late O-type for the brightest few objects). The location of these sources in the CMDs (Fig. 3) provides support for our classifications in most cases.

For four spectra with $S / N>10$ and well-defined $\mathrm{H} \beta$ absorption (and without obvious evidence for nebular oversubtraction) we estimated radial velocities (RV) from Gaussian fits to the observed $\mathrm{H} \beta$ absorption (included in last column of Table 1$)$. The weighted-mean velocity $(\overline{\mathrm{RV}})$ from the four spectra is $\overline{\mathrm{RV}}=255 \pm 13 \mathrm{~km} \mathrm{~s}^{-1}$. The velocity of the He II $\lambda 4686$

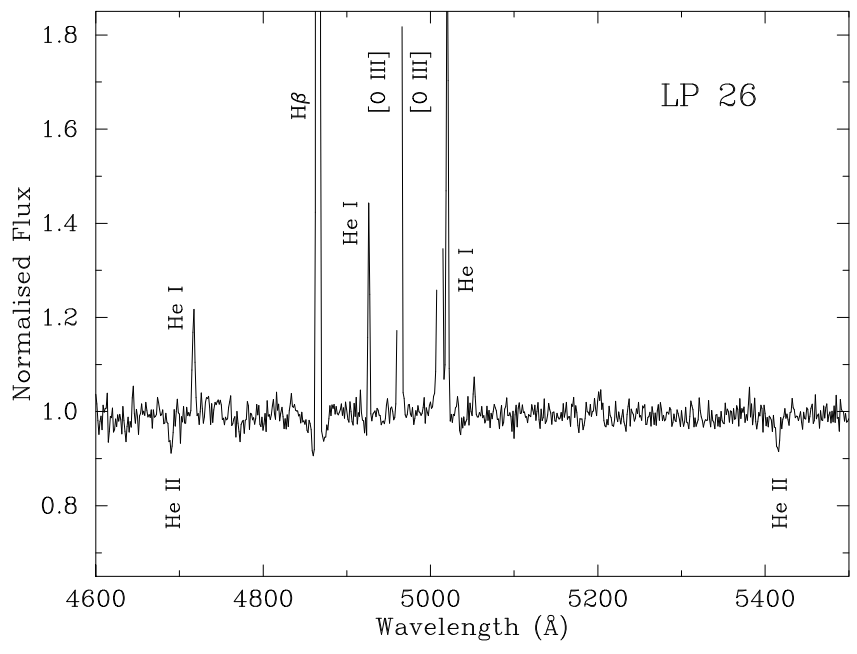

Fig. 4. Blueward part of the MUSE spectrum of the extracted central source of the H II region in Leo P (LP 26). The identified emission lines, from left-to-right by species, are: $\mathrm{H} \beta$; $\mathrm{He}$ I $\lambda \lambda 4713,4922,5016$; [O III] $\lambda \lambda 4959,5007$. The He II $\lambda \lambda 4686,5411$ absorption lines provide the first direct evidence for an O-type star in Leo P.

absorption in the spectrum of LP 26 in the H II region is consistent with these values $\left(252 \pm 35 \mathrm{~km} \mathrm{~s}^{-1}\right)$. Given the relatively large uncertainties on the MUSE values, they are in good agreement with the systemic velocity of neutral hydrogen of $v_{\text {LSRK }}=260.8 \pm 2.5 \mathrm{~km} \mathrm{~s}^{-1} \quad$ (Bernstein-Cooper et al. 2014), which equates to a heliocentric velocity of approx. $265 \mathrm{~km} \mathrm{~s}^{-1}$ in the direction of Leo P.

\subsection{Cool stars}

Near-infrared imaging of Leo $\mathrm{P}$ was used by Lee (2016) to identify 22 candidate AGB stars, six of which lie within the MUSE field. The spectra of five of these are shown in Fig. 6, with their observational properties summarised in Table 2; the sixth (MSB \#56) is a background galaxy (see Table 3). The two brightest candidates are carbon stars, as revealed by the strong $\mathrm{C}_{2}$ Swan bandheads at $\lambda \lambda 5165,5636$. We compared the wavelengths of the bandheads in the MUSE spectra to those in an archival UVES spectrum from Bagnulo et al. (2003) of the Galactic carbon star, W Ori (HD 32736), finding differential velocities that are consistent with their membership of Leo P (i.e. $\delta \mathrm{RV} \sim 250 \mathrm{~km} \mathrm{~s}^{-1}$ ).

The remaining three stars, suggested as oxygen-rich M-type AGB stars by Lee, are relatively featureless. However, there appears to be absorption in the near-IR Ca II Triplet (CaT, with rest wavelengths of $\lambda \lambda 8498,8542,8662)$ for MSB 13. An expanded view of the $\mathrm{CaT}$ region for the candidate AGB sources is shown in Fig. 7, with absorption also present for $\lambda 8542$ in MSB 18. Indeed, of the three CaT lines in the MUSE data, the central $\lambda 8542$ line is the most robust in terms of being less influenced by sky residuals. From a Gaussian fit to the $\lambda 8542$ line in MSB 13 we estimated RV $=262 \pm 8 \mathrm{~km} \mathrm{~s}^{-1}$, in good agreement with the values for the hotter stars. To investigate the $\mathrm{CaT}$ region for the fainter stars in the RGB, the lower spectrum in Fig. 7 shows the co-added data of the 16 sources with $F 814 \mathrm{~W} \leq 23.0$. The stronger $\mathrm{CaT}$ components can again be seen, confirming the presence of cool, evolved stars as expected from the CMD (Fig. 3).

Thus, we conclude that at least two of these candidate AGB stars appear to be cool, evolved stars with CaT 


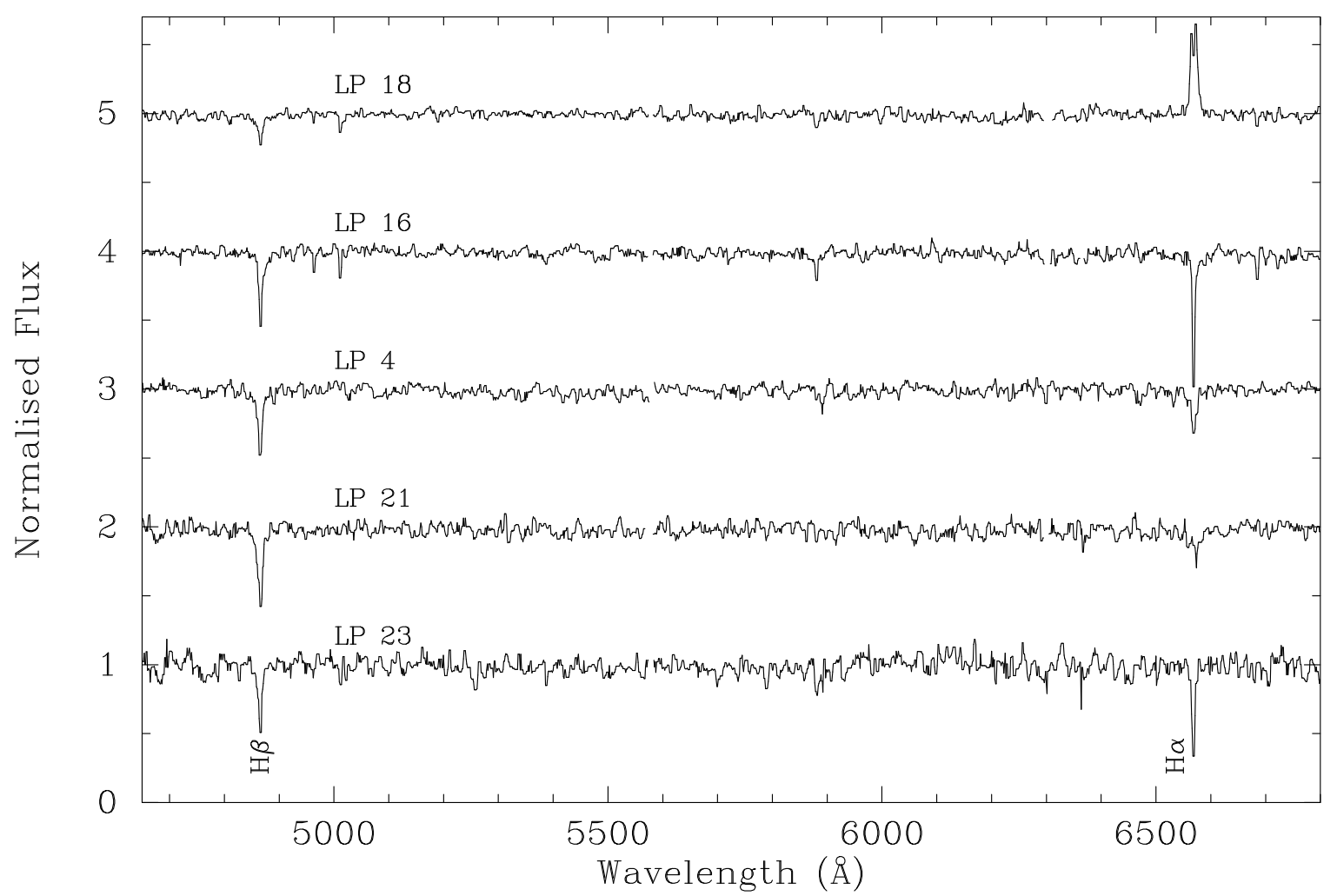

Fig. 5. Best five examples of spectra of presumed BA-type stars in Leo P (each smoothed using a five-pixel boxcar filter). In addition to the Balmer line absorption, there is tentative detection of He I $\lambda \lambda 5876,6678$ absorption in LP 16 and LP 18, although potentially related to the apparent over-subtraction of nebular features (as demonstrated by the [O III] $\lambda \lambda 4959,5007$ "absorption" and similar evidence in the cores of the $\mathrm{H} \alpha$ lines).



Fig. 6. MUSE spectra of candidate AGB stars from Lee (2016), in which each spectrum was smoothed using a five-pixel boxcar filter and displaced vertically by increasing multiples of 500 counts. The expected location of absorption by the Ca II triplet is indicated for MSB 13 , with an expanded view of this region shown in Fig. 7. Mean counts over the 8110-8270 ̊ range (which is relatively free of sky residuals) for the five spectra are, from top to bottom, 396, 343, 314, 171, 125, in keeping with the $I$-band and $F 814 W$ magnitudes in Table 2. 
Table 1. Confirmed and candidate blue stars in the Leo P MUSE field.

\begin{tabular}{|c|c|c|c|c|c|c|c|c|c|}
\hline LP\# & MSB & $\alpha(\mathrm{J} 2000)$ & $\delta(\mathrm{J} 2000)$ & $V$ & $I$ & $F 475 W$ & $F 814 W$ & $S / N$ & Comments \\
\hline 1 & 138 & 102143.66 & +180528.50 & 24.40 & 23.90 & 24.459 & 24.087 & 4 & Candidate \\
\hline 2 & 207 & 102143.71 & +180521.69 & 24.79 & 24.64 & 24.737 & 24.819 & 5 & Candidate \\
\hline 3 & 94 & 102143.89 & +180519.35 & 23.98 & 23.68 & 24.156 & 23.880 & 7 & Candidate \\
\hline 4 & 16 & 102143.92 & +180556.59 & 22.50 & 22.29 & 22.521 & 22.561 & 19 & $\mathrm{RV}(\mathrm{H} \beta)=240 \pm 19 \mathrm{~km} \mathrm{~s}^{-1}$ \\
\hline 5 & 202 & 102143.97 & +180519.29 & 24.83 & 24.09 & 24.973 & 24.046 & 4 & Candidate \\
\hline 6 & 40 & 102144.01 & +180523.81 & 23.45 & 23.08 & 23.707 & 23.348 & 8 & \\
\hline 7 & 98 & 102144.37 & +180529.45 & 24.09 & 23.52 & 24.613 & 24.431 & 5 & Candidate \\
\hline 8 & $\ldots$ & 102144.39 & +180525.31 & $\ldots$ & $\ldots$ & 24.419 & 24.318 & 9 & \\
\hline 9 & 92 & 102144.47 & +180525.62 & 23.95 & 23.66 & 24.111 & 23.961 & 6 & Candidate \\
\hline 10 & $\ldots$ & 102144.55 & +180520.00 & $\ldots$ & $\ldots$ & 23.958 & 24.013 & 9 & Candidate \\
\hline 11 & $\ldots$ & 102144.63 & +180516.14 & & .. & 23.834 & 23.908 & 4 & Candidate \\
\hline 12 & $\ldots$ & 102144.69 & +180521.07 & & .. & 24.363 & 24.488 & 7 & Candidate \\
\hline 13 & $\ldots$ & 102144.69 & +180523.47 & $\ldots$ & $\ldots$ & 24.090 & 24.155 & 9 & Candidate \\
\hline 14 & 25 & 102144.76 & +1805 27.17 & 23.14 & 23.00 & 23.276 & 23.421 & 10 & $\mathrm{Be} / \mathrm{Ae}$ ? (H $\alpha$ em.?) \\
\hline 15 & $\ldots$ & 102144.79 & +180525.15 & $\ldots$ & $\ldots$ & 24.251 & 24.540 & 7 & Candidate \\
\hline 16 & 15 & 102144.81 & +180522.31 & & & 22.790 & 23.021 & 21 & \\
\hline 17 & $\ldots$ & 102144.89 & +180524.33 & $\ldots$ & $\ldots$ & 24.634 & 24.850 & 8 & \\
\hline 18 & 12 & 102144.90 & +180522.38 & 22.16 & 22.02 & 22.255 & 22.304 & 28 & $\mathrm{RV}(\mathrm{H} \beta)=274 \pm 19 \mathrm{~km} \mathrm{~s}^{-1}$ \\
\hline 19 & 41 & 102144.95 & +180506.28 & 23.46 & 23.37 & 23.483 & 23.497 & 8 & \\
\hline 20 & $\ldots$ & 102144.97 & +180524.72 & $\ldots$ & $\ldots$ & 24.768 & 24.511 & 6 & Candidate \\
\hline 21 & $\ldots$ & 102145.00 & +180525.59 & $\ldots$ & $\ldots$ & 22.579 & 22.568 & 15 & $\mathrm{RV}(\mathrm{H} \beta)=260 \pm 22 \mathrm{~km} \mathrm{~s}^{-1}$ \\
\hline 22 & 24 & 102145.01 & +180513.21 & 23.15 & 22.87 & 23.369 & 23.308 & 11 & $\mathrm{Be} / \mathrm{Ae} ?(\mathrm{H} \alpha \& \mathrm{H} \beta$ em.?) \\
\hline 23 & $\ldots$ & 102145.03 & +180524.80 & $\ldots$ & $\ldots$ & 22.473 & 22.585 & 12 & $\mathrm{RV}(\mathrm{H} \beta)=247 \pm 20 \mathrm{~km} \mathrm{~s}^{-1}$ \\
\hline 24 & $\ldots$ & 102145.03 & +180525.02 & $\ldots$ & $\ldots$ & 23.066 & 23.052 & 3 & Candidate \\
\hline 25 & $\cdots$ & 102145.08 & +180516.35 & & & 24.290 & 24.684 & 15 & On SE edge of $\mathrm{H}$ II region \\
\hline 26 & 2 & 102145.10 & +180516.62 & $\ldots$ & $\ldots$ & 21.464 & 21.837 & 52 & O-type star in $\mathrm{H}$ II region \\
\hline 27 & 82 & 102145.12 & +180506.92 & 23.92 & 23.36 & 24.322 & 23.740 & 5 & Candidate \\
\hline 28 & 22 & 102145.13 & +180513.76 & 23.01 & 23.16 & 22.999 & 23.349 & 13 & Candidate \\
\hline 29 & 33 & 102145.14 & +180520.31 & 23.27 & 23.09 & 23.480 & 23.465 & 7 & \\
\hline 30 & 63 & 102145.21 & +180509.09 & 23.73 & 23.41 & 24.435 & 24.488 & 8 & \\
\hline 31 & 114 & 102145.26 & +180529.82 & 24.17 & 23.89 & 24.294 & 24.276 & 4 & Candidate \\
\hline 32 & 106 & 102145.27 & +180507.84 & 24.11 & 23.90 & 24.238 & 24.080 & 5 & Candidate \\
\hline
\end{tabular}

Notes. Identifiers in the second column and $V$ - and $I$-band photometry are from (MSB, McQuinn et al. 2013); $F 475 W$ and $F 814 W$ photometry is from the HST imaging from McQuinn et al. (2015). S/N estimates (per pixel) were obtained over the 5050-5400 $\AA$ part of the normalised spectra.

Table 2. Confirmed and candidate cool stars in the MUSE field.

\begin{tabular}{lccccccccll}
\hline \hline MSB & $\alpha(\mathrm{J} 2000)$ & $\delta(\mathrm{J} 2000)$ & $V$ & $I$ & $F 475 W$ & $F 814 W$ & $J$ & $K$ & Classification & Comments \\
\hline 18 & 102142.33 & +180546.87 & 23.27 & 21.70 & 24.129 & 21.823 & 20.972 & 20.254 & AGB? & Cand. M-AGB (L16) \\
10 & 102143.85 & +180530.70 & 23.00 & 20.97 & 24.175 & 21.029 & 20.073 & 18.773 & Carbon star & Cand. C-AGB (L16) \\
8 & 102144.24 & +180546.50 & 22.97 & 20.90 & 24.099 & 20.878 & 19.464 & 18.451 & Carbon star & Cand. M-AGB (L16) \\
13 & 102144.83 & +180530.87 & 22.63 & 21.20 & 23.430 & 21.308 & 20.495 & 19.691 & AGB? & Cand. M-AGB (L16) \\
21 & 102144.73 & +180548.13 & 23.30 & 21.97 & 23.959 & 22.040 & 21.198 & 20.311 & $?$ & Cand. M-AGB (L16) \\
\hline 1 & 102143.27 & +180513.36 & 20.02 & 20.03 & $\ldots$ & $\ldots$ & 15.692 & 14.979 & M (f/g?) & 2MASS: J10214334+1805133 \\
$\ldots$ & 102143.32 & +180503.22 & $\ldots$ & $\ldots$ & $\ldots$ & $\ldots$ & 16.156 & 15.362 & K (f/g) & 2MASS: J10214332+1805032 \\
5 & 102143.48 & +180509.43 & 22.05 & 20.27 & 22.975 & 19.796 & $\ldots$ & $\ldots$ & M (f/g) & \\
\hline
\end{tabular}

Notes. Identifiers and $V$ - and $I$-band photometry are from McQuinn et al. (2013); F475W and $F 814 W$ photometry is from HST imaging by McQuinn et al. (2015). IR photometry is from Lee (2016, with his classifications in the final column), and 2MASS (Skrutskie et al. 2006) for two of the foreground objects.

absorption (and with $F 814 W$ magnitudes that are brighter than the TRGB). The corresponding lack of strong molecular bands (e.g. from $\mathrm{TiO}$ ) in their spectra in Fig. 6 is notable compared with what is usually seen in (M-type) AGB specta, but not unexpected given the low metallicity of Leo $\mathrm{P}$ (as traced by the published oxygen abundance). Indeed, the ratio of C-rich to M-type (O-rich) AGB stars is known to be a function of metallicity, with an increasing $\mathrm{C} / \mathrm{M}$ ratio towards lower metallicities (e.g. Iben \& Renzini 1983; Battinelli \& Demers 2005). The relative dearth of M-type objects at low metallicities is attributed to the quicker dredge-up timescales to become a carbon star, combined with higher temperatures from the evolutionary models, which would act to reduce the $\mathrm{TiO}$ absorption, hence fewer M-type spectra (see, e.g., discussion by Kacharov et al. 2012). 


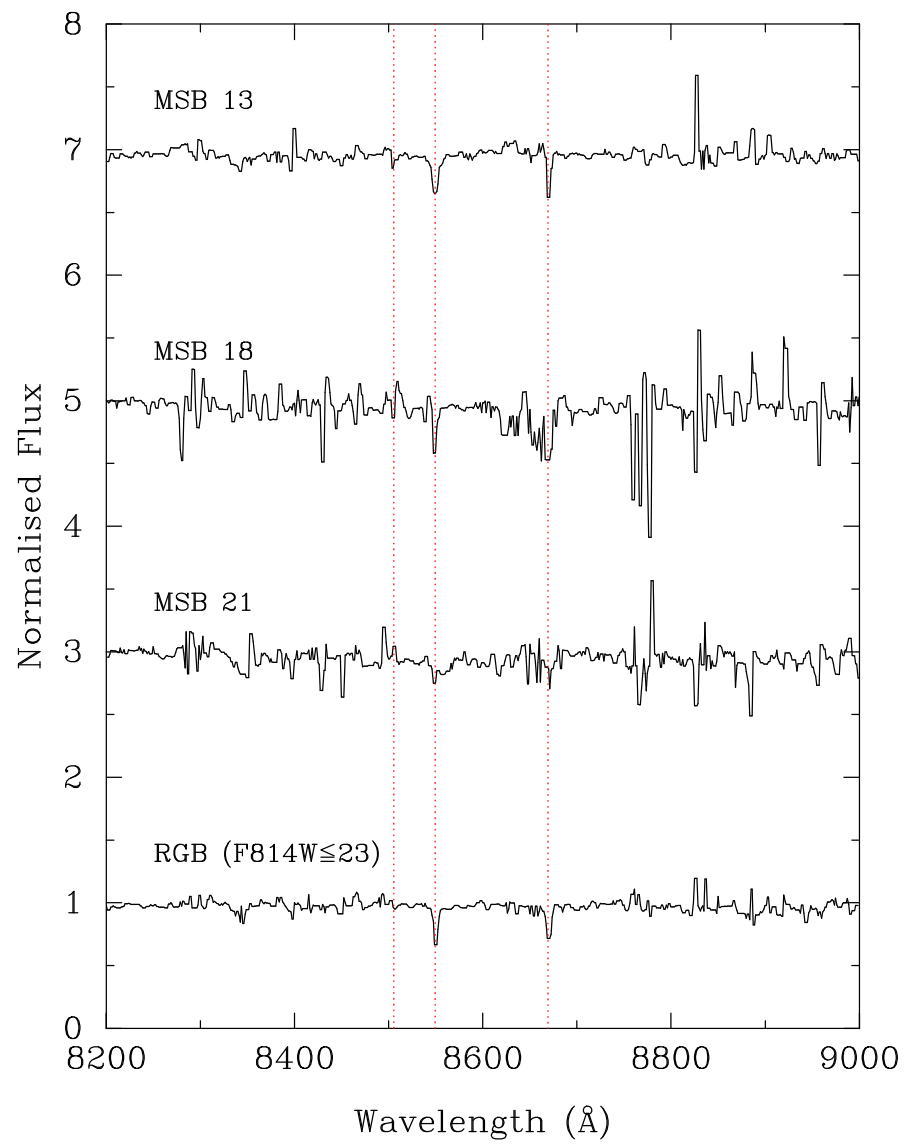

Fig. 7. Region around the $\mathrm{Ca}$ II triplet (CaT) for the three stars previously identified as candidate AGB stars, together with a stacked spectrum of the 16 next brightest objects at the top of the red giant branch (i.e. excluding the three candidate AGB stars). CaT absorption is seen in the brightest two (MSB 13 and 18) and in the stacked data.

Equally, it is plausible that simple metallicity effects give the impression of earlier spectral types, even if the temperatures were not that different. For example, a luminous, cool star with an effective temperature of $4000 \mathrm{~K}$ would be classified as Mtype in the LMC, but at the metallicity of Leo P, the TiO bands would weaken sufficiently that it would be classified as a K-type spectrum.

All five of the objects from Lee (2016) are relatively isolated, point-like sources in the HST imaging, and we highlight that four are somewhat removed from the central part of the system. This suggests the intermediate-age population is either more extended or well mixed, with the latter similar to the findings for six of the nine dwarf galaxies studied by McQuinn et al. (2017). Following the arguments of McQuinn et al. (2017), we note that MSB 18 is at a distance of $50^{\prime \prime}$ (equivalent to $400 \mathrm{pc}$ ) from the $\mathrm{H}$ II region, potentially providing a source of chemical enrichment in the outer regions of Leo $\mathrm{P}$.

\subsection{Foreground stars}

There are three seemingly foreground stars included in our extractions from the MUSE cube, as listed at the end of Table 2. One star (MSB 1) has a somewhat larger RV than might be expected for a foreground Galactic object. Cross-correlation of the two M-type spectra (over the $\lambda \lambda 4600-5565$ range) yielded a differential RV of $168 \pm 20 \mathrm{~km} \mathrm{~s}^{-1}$, in good agreement with absolute estimates from the CaT of $172 \pm 6 \mathrm{~km} \mathrm{~s}^{-1}$.

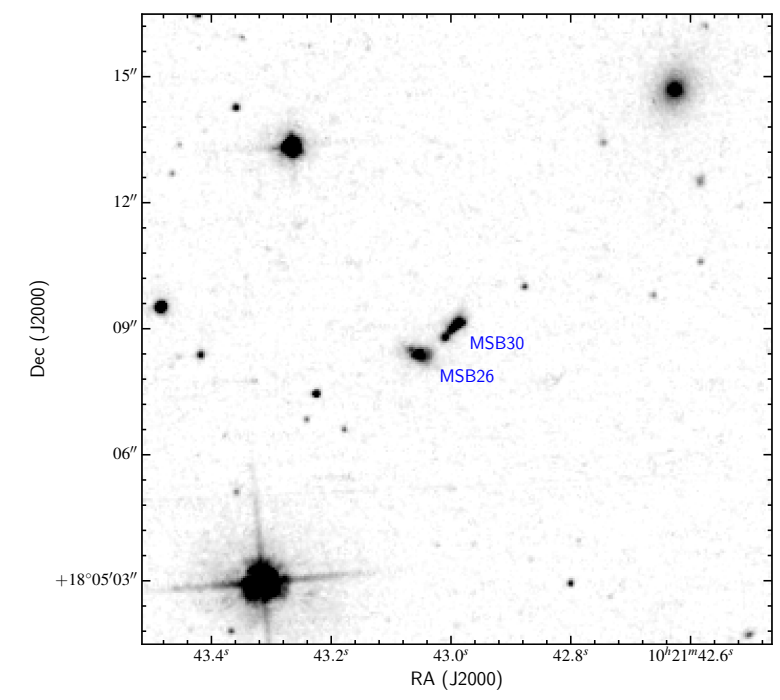

Fig. 8. Two $z \sim 2.5$ sources in the HST-ACS $F 475 W$ image from McQuinn et al. (2015). At the resolution of HST, the extracted MUSE sources are both resolved into two components.

\subsection{Background galaxies}

From initial extractions of the MUSE sources using source detections and simple aperture extractions, we found 20 sources which are background galaxies. Indeed, a number of these are clearly visible as such in the HST imaging (see discussion of two examples by Lee 2016), meaning that they are not included in the CMD in Fig. 3 as they were rejected as non-stellar sources in the photometric analyis. Observational details for the spectroscopically-confirmed galaxies are given in Table 3, including estimates of their redshifts $(z)$ using the diagnostic emission or absorption features as indicated. Of note are the two systems at $z=2.5$, located adjacent to each other on the sky. The HST imaging reveals that both MUSE sources are comprised of two components, as shown in Fig. 8.

\section{Expected absolute magnitudes at low $Z$}

We could potentially use the estimated absolute magnitude of LP 26 to (coarsely) constrain its spectral type, but we are unfortunately limited to Galactic results (e.g. Walborn 1972; Wegner 2000; Martins et al. 2005). To investigate the potential impact of the very low metallicity in a system like Leo P on the expected stellar parameters we turned to evolutionary models calculated by Szécsi et al. (2015) for IZw 18 (with $Z=0.02 Z_{\odot}$ ). Figure 9 shows the zero-age main sequence (ZAMS) as traced by their non-rotating models compared to results for the Galaxy, LMC and Small Magellanic Cloud (SMC) from Brott et al. (2011); for completeness the LMC tracks are supplemented by results for $M>60 M_{\odot}$ from Köhler et al. (2015). Also shown in Fig. 9 are the ZAMS positions for models with initial masses of 9, $10,15,20$, and $30 M_{\odot}$, highlighting the temperature dependence vs. metallicity for stars of a given mass (at near constant luminosity, corresponding to smaller radii at lower metallicities); similar differences are also seen in the rotating models from the same grids. A shift in the same sense is also seen in the "Geneva" evolutionary models, for example the comparison in Fig. 20 from Szécsi et al. of the ZAMS for the SMC-like models of Georgy et al. (2013) and the near-zero metallicity models of Meynet \& Maeder (2002).

As an example case, consider the ZAMS temperatures in Fig. 9 for a $20 M_{\odot}$ star where $T_{\text {eff }}(\mathrm{MW})=35.3 \mathrm{kK}$ cf. 
Table 3. Background galaxies in the MUSE field.

\begin{tabular}{lcccccl}
\hline \hline MSB & $\alpha(\mathrm{J} 2000)$ & $\delta(\mathrm{J} 2000)$ & $V_{\mathrm{o}}$ & $I_{\mathrm{O}}$ & $z$ & Diagnostic \\
\hline 19 & 102142.43 & +180517.38 & 23.41 & 21.70 & 0.5436 & [O II] $\lambda 3727$ \\
39 & 102142.46 & +180542.80 & 23.88 & 22.33 & 0.7543 & [O II] $\lambda 3727$ \\
56 & 102142.55 & +180547.16 & 24.62 & 22.53 & 0.5169 & [O II] $\lambda 3727$ \\
37 & 102142.56 & +180517.56 & 24.19 & 22.24 & 0.7554 & [O II] $\lambda 3727$ \\
4 & 102142.62 & +180514.59 & 21.95 & 20.26 & 0.3925 & Ca $H+K$ \\
62 & 102142.87 & +180533.13 & 24.15 & 22.64 & 0.7539 & [O II] $\lambda 3727$ \\
48 & 102142.97 & +180538.43 & 24.22 & 22.40 & 0.7549 & [O II] $\lambda 3727$ \\
30 & 102142.99 & +180508.98 & 23.30 & 22.62 & 2.5007 & Near-UV Fe II, Mg II \\
49 & 102143.01 & +180559.13 & 23.98 & 22.50 & 0.8883 & [O II] $\lambda 3727$ \\
26 & 102143.04 & +180508.34 & 23.29 & 22.36 & 2.5005 & Near-UV Fe II, Mg II \\
7 & 102143.10 & +180517.26 & 23.07 & 20.88 & 0.5440 & [O II] $\lambda 3727$ \\
31 & 102143.39 & +180538.07 & 23.60 & 22.25 & 0.7462 & [O II] $\lambda 3727$ \\
17 & 102144.15 & +180503.11 & 22.84 & 21.82 & 0.4763 & [O II] $\lambda 3727$ \\
108 & 102144.15 & +180511.12 & 24.29 & 23.23 & 0.8320 & [O II] $\lambda 3727$ \\
- & 102144.44 & +180505.53 & $\ldots$ & $\ldots$ & 0.4763 & [O II] $\lambda 3727$ \\
168 & 102145.28 & +180534.77 & 24.57 & 24.17 & 0.3603 & [O II] $\lambda 3727$ \\
151 & 102145.68 & +180538.30 & 25.00 & 23.38 & 0.6028 & [O II] $\lambda 3727$ \\
172 & 102146.04 & +180535.46 & 24.78 & 23.67 & 0.8149 & [O II] $\lambda 3727$ \\
163 & 102146.05 & +180549.46 & 24.86 & 23.52 & 0.9692 & [O II] $\lambda 3727$ \\
- & 102146.18 & +180559.60 & $\ldots$ & $\ldots$ & 0.4137 & Ca $H+K$ \\
\hline
\end{tabular}

Notes. Identifiers and magnitudes are from McQuinn et al. (2013). MSB 56 was previously identified as a candidate C-AGB star by Lee (2016). Typical uncertainties on the estimated redshifts are \pm 0.0002 , except for \#26 and 30, where the dispersion of the seven near-UV lines (Fe II $\lambda \lambda 2343$, 2374, 2382, 2586, 2599; $\mathrm{Mg}$ II $\lambda \lambda 2796,2803)$ was \pm 0.0005 .

$T_{\text {eff }}(\mathrm{IZw} 18)=41.2 \mathrm{kK}$. The models at the ZAMS have (near) constant luminosities, but such stars will have different bolometric corrections (BCs) due to the increasing temperatures towards lower metallicity. To investigate the scale of this effect we highlight the BCs from the TLUSTY OSTAR2002 grids (Lanz \& Hubeny 2003). A Galactic model for $35 \mathrm{kK}$ has $\mathrm{BC}=-3.24 \mathrm{mag}$, cf. $\mathrm{BC} \sim-3.8 \mathrm{mag}$ for the higher temperature at $Z=0.03 Z_{\odot}$ (interpolating between $T_{\text {eff }}=40$ and $42.5 \mathrm{kK})$.

There is a similarly large difference for the $9 M_{\odot}$ models, ranging from $24.5 \mathrm{kK}$ in the Galaxy to $30 \mathrm{kK}$ for IZw 18 . We again turned to the TLUSTY results to assess the potential impact of this on the $\mathrm{BC}$ values, with $\mathrm{BC} \sim-2.95$ for the hotter, metal-poor star. The cooler, Galactic temperature is beyond the OSTAR2002 grid, but an estimate of BC $\sim-2.40$ is available from the companion BSTAR2006 grid (Lanz \& Hubeny 2007). We require similar calculations with the latest wind codes but these results illustrate that, for a given mass, the visual absolute magnitudes of high-mass stars at such low metallicities could be $\sim 0.5$ mag fainter than we would otherwise expect (together with an increase in the number of ionising photons). Alternatively, this also implies that fewer massive stars could potentially account for a given level of ionisation in unresolved systems at very low- $Z$.

We are currently unable to link initial mass to spectral type as a function of metallicity, but the differences above echo the effect where metallicity is known to impact on the temperature scale of OB-type stars of a given spectral type (e.g. Massey et al. 2005; Mokiem et al. 2006, 2007; Trundle et al. 2007). At lower metallicities the cumulative opacity of the metal lines is diminshed, resulting in less "back warming" by trapped radiation and hotter models are required to reproduce the observed ratios of spectral lines (also see discussion by Martins et al. 2005).

Taking this into consideration for the calibrations from Martins et al. (2005), the estimated absolute visual magni-

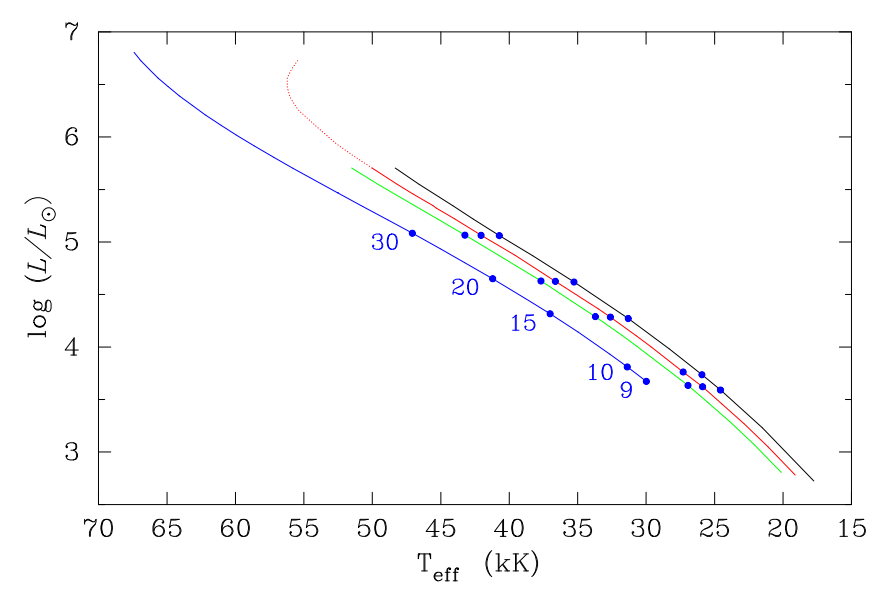

Fig. 9. Zero-age main sequence in the Hertzsprung-Russell diagram for (non-rotating) evolutionary models with $Z=0.02 Z_{\odot}$ (blue, Szécsi et al. 2015), and for the SMC (green), LMC (red), and Galaxy (black) from Brott et al. (2011); the LMC results are supplemented by higher-mass models (dotted red line) from Köhler et al. (2015). Results for five initial masses (in units of $M_{\odot}$ ) are highlighted by the blue dots to illustrate the temperature dependence vs. metallicity (but with no $10 M_{\odot}$ model available for the SMC).

tude from McQuinn et al. $\left(M_{V}=-4.43\right)$ constrains the ionising source in the $\mathrm{H}$ II region in Leo P to a mid O-type star. Given the prevalence of binarity in massive stars, McQuinn et al. argued that this source could perhaps be two $\mathrm{O} 7$ or $\mathrm{O} 8$ stars, with stellar masses of $\sim 25 M_{\odot}$. Such a mass exceeds the maximum (of 2.5-3 $M_{\odot}$ ) expected at the low star-formation rate of Leo $\mathrm{P}$ from the integrated galactic initial mass function (IGIMF) approach (Pflamm-Altenburg et al. 2007). Aside from the specific physical properties of LP 26, its spectroscopic confirmation as an O-type star supports the conclusions of McQuinn et al. in this regard, 


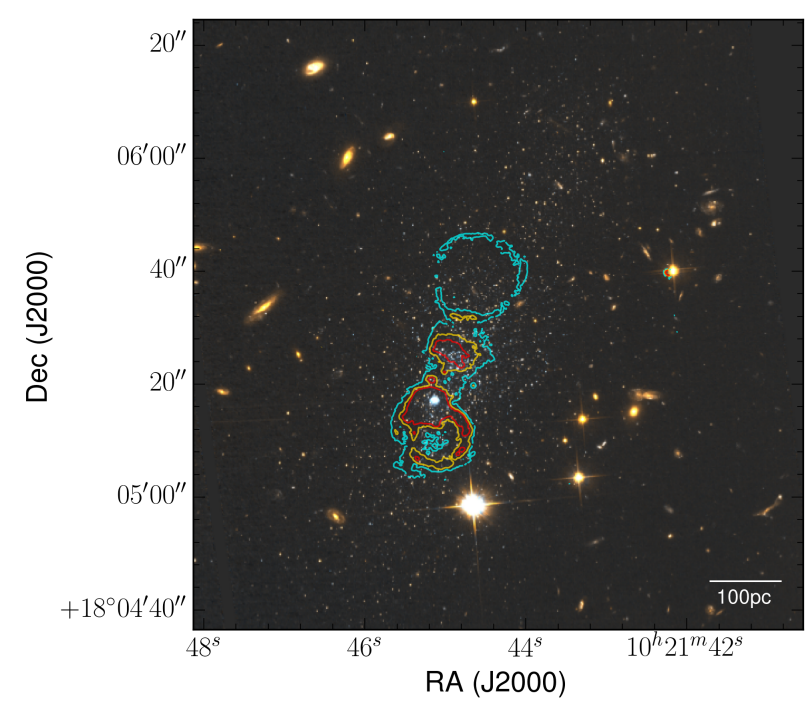

Fig. 10. $\mathrm{H} \alpha$ emission in Leo $\mathrm{P}$ overlaid on the combined $F 475 W+$ $F 814 W$ HST image (cyan, yellow and red contours are $\mathrm{H} \alpha$-emission levels of 5,10 , and $15 \times 10^{-20} \mathrm{erg} \mathrm{s}^{-1} \mathrm{~cm}^{-2}$, respectively). The known $\mathrm{H}$ II region is the compact blue source in the main southern feature. The MUSE data have also revealed a region of $\mathrm{H} \alpha$ emission just to the north, and two large extended shells to the south and (far) north (see also Fig. 11).

i.e. that the upper limit to the IMF - even if sparsely sampled is not so significantly influenced by the star-formation rate. More recently, Jeřábková et al. (2018) have argued that the inferred star-formation rate in the IGIMF theory for Leo $\mathrm{P}$ would be significantly larger than the value estimated from the current $\mathrm{H} \alpha$ luminosity of the $\mathrm{H}$ II region; this helps to reconcile the predictions with the detection of the O-type star.

\section{Nebular emission}

Inspecting the MUSE data at the wavelength of $\mathrm{H} \alpha$ emission we discovered three new structures compared to the original discovery images, suggesting more than one site of (relatively) recent star formation, as shown in the contours in Fig. 10 and the upper panels of Fig. 11. Part of the southern shell is also traced by $\mathrm{H} \beta$ emission (middle panels), while [O III] $\lambda 5007$ emission is only seen in the vicinity of the $\mathrm{H}$ II region (lower panels). Intensity maps of [O III] $\lambda 4959,[\mathrm{~N} \mathrm{II}] \lambda \lambda 6584,6548$, and [S II] $\lambda \lambda 6717$, 6731 were comparable to that for [O III] $\lambda 5007$, in the sense that the large structures seen in $\mathrm{H} \alpha$ are absent (perhaps simply linked to the low metallicity).

The northern $\mathrm{H} \alpha$ shell has an apparent diameter of $\sim 15^{\prime \prime}$. Adopting a distance of $1.62 \mathrm{Mpc}$ (McQuinn et al. 2015), this is equivalent to a projected diameter of $\sim 120 \mathrm{pc}$. The northern and southern shells appear to be fairly well defined rings of emission, whereas a central shell appears somewhat more diffuse. The H II region is located on northern edge of the southern ring.

The positions of our spectroscopically-confirmed stars are overlaid in the left-hand panels of Fig. 11. The absence of massive stars in the northern shell suggests this is an older formation (e.g. supernova remnant) rather than a more recent wind-blown bubble. Only two of our sources lie within the southern ring, including LP22, one of the candidate Be/Ae stars, which is unlikely to be the driving source for such a ring, again suggesting it as a supernova remnant. Indeed, given the large number of high-mass x-ray binaries with Be-type components in the low-metallicity environment of the SMC (e.g. Coe et al
2005), we speculate that LP 22 warrants further study to investigate for the presence of a degenerate companion. Lastly, we also note the four stars along the southern edge of the southern ring, suggestive of a connection in their formation.

Using a similar profile-fitting approach to that used by Castro et al. (2018) for MUSE observations of 30 Doradus, velocity maps for each emission line are shown in the centre panels of Fig. 11 (in which the data are spatially smoothed with a Gaussian function with a spatial FWHM of $0.6^{\prime \prime}$ prior to the fits). We also investigated maps of the standard deviation $(\sigma)$ of the $\mathrm{H} \alpha$ Gaussian fits to investigate the velocity dispersion of the gas, but these do not add much to the velocity maps, aside from hints of a slightly larger dispersion in the northern shell.

The systemic velocity of the gas $\left(\sim 265 \mathrm{~km} \mathrm{~s}^{-1}\right)$ is in good agreement with the $\mathrm{H}$ I and stellar velocities. There appears to be a small velocity gradient of a few $\mathrm{km} \mathrm{s}^{-1}$ across the $\mathrm{H}$ II region in the $\mathrm{H} \beta$ map, with a similar trend in [O III], but given the limitations of the MUSE data, we defer detailed study of the kinematics to future observations at higher spectral resolution (e.g. with the Keck Cosmic Web Imager, Morrissey et al. 2018).

Prompted by the discovery of these spatially-distinct structures we re-examined the HST photometry of all the stars in the four regions (i.e. including those without spectroscopic confirmation from MUSE). This led to relatively sparse samples and aside from reinforcing the relative dearth of luminous, blue stars in the northern and southern regions, we were unable to glean further insights into the histories of these regions.

\section{Concluding remarks}

We have presented the first stellar spectroscopy in Leo P, a low luminosity, dwarf galaxy at a distance of $1.62 \pm 0.15 \mathrm{Mpc}$. Our findings from the MUSE observations include:

- Spectroscopic confirmation of an O-type star (LP 26) in the $\mathrm{H}$ II region via observations of He II absorption. From consideration of its absolute magnitude (and assuming the published distance), this is probably a mid O-type star (Fig. 4).

- Fourteen sources with $\mathrm{H} \beta$ absorption and rising blue flux distributions that confirms them as hot stars. Given the faintness of these targets $(22<V<25)$ we were unable to comment more on their spectral classifications, but from their locations in the CMD we suggest these are B-type (or late O-type) objects (Fig. 5). We tentatively classify a further 17 sources as candidate hot stars, again via detection of $\mathrm{H} \beta$ absorption.

- Confirmation of two candidate AGB stars from Lee (2016) as carbon stars, and confirmation of two further candidates as luminous cool (presumably AGB) stars via detection of CaT absorption in their spectra (Figs. 6 and 7).

- Confirmation of CaT absorption in the RGB population, via co-adding the spectra of the brightest members (Fig. 7).

- Two $100 \mathrm{pc}$-scale ring structures that are traced by $\mathrm{H} \alpha$ emission from the gas, with the $\mathrm{H}$ II region located on the northern edge of the southern ring (Fig. 11).

In addition, to investigate the expected temperatures and magnitudes of massive stars in Leo P, we employed evolutionary tracks from Szécsi et al. (2015) for single stars at the (near identical) metallicity of the IZw 18 galaxy. The shift of the ZAMS to higher temperatures with decreasing metallicity has long been known, but we argue that the significance of this effect at very low metallicites could impact on the apparent magnitudes of massive stars. For a given stellar luminosity, hotter temperatures give larger bolometric corrections, which would mean that stars of a comparable mass in Leo P or IZw 18 will have fainter absolute visual magnitudes than in the Galaxy (by $\sim 0.5 \mathrm{mag}$ 

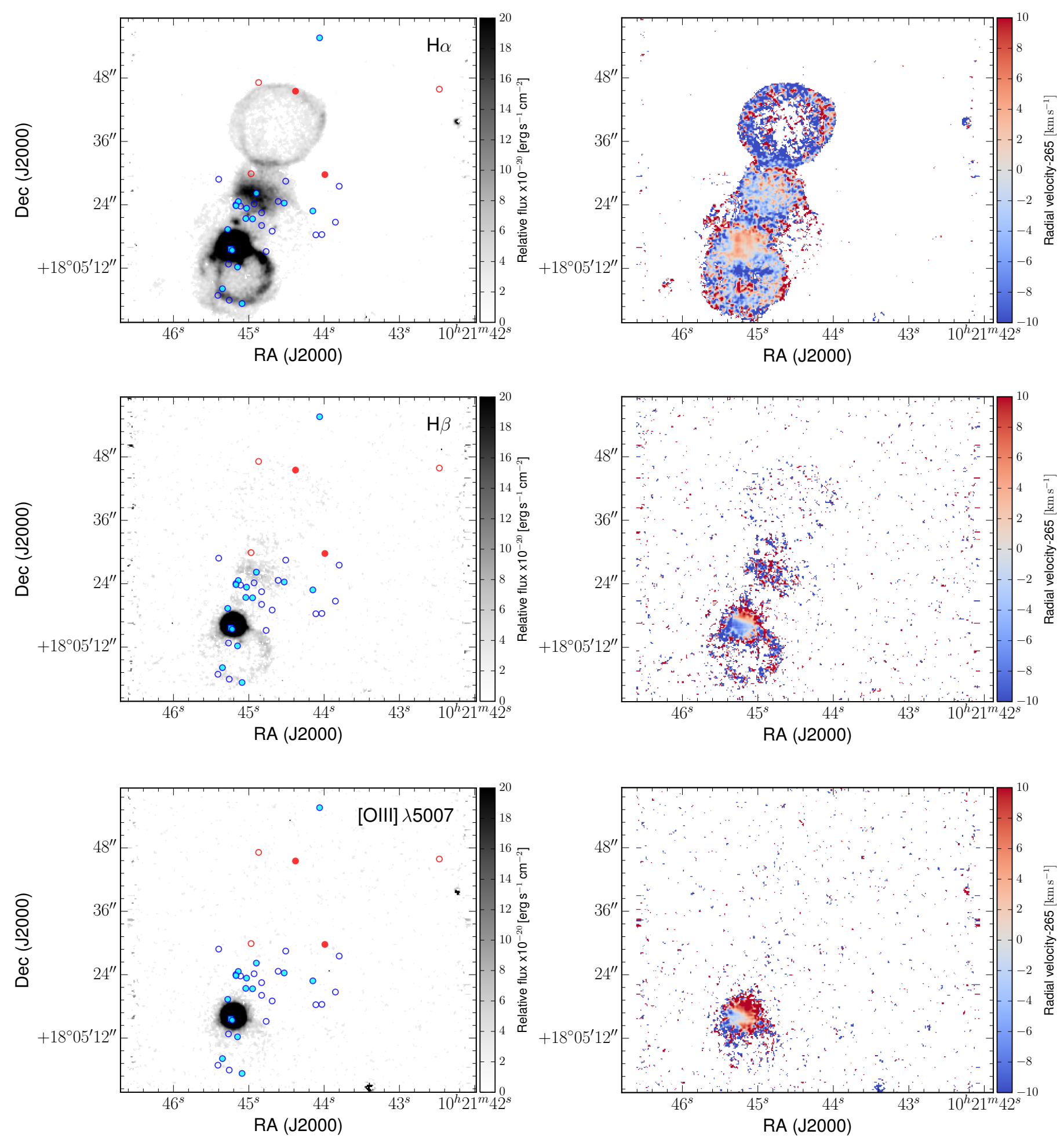

Fig. 11. Intensity and velocity maps for the ionised gas in Leo P. The stars classified from the MUSE spectra are overplotted using the same symbols as in Fig. 2, i.e.: massive stars (filled cyan circles), candidate massive stars (open blue circles), carbon stars (filled red circles), additional candidate AGB stars (open red circles). Upper panels: $\mathrm{H} \alpha$ emission; middle panels: $\mathrm{H} \beta$; lower panels: [O III] $\lambda 5007$. Left-hand panels: intensity of the given emission line; right-hand panels: differential velocities from Gaussian fits to the observed lines.

in the example cases of the 9 and $20 M_{\odot}$ models considered here).

While giving us our first tantalysing view of high-mass stars in a very metal-poor environment, the upper mass function is so sparsely populated that we were not able to test the predictions of Szécsi et al. (2015) regarding core-hydrogen- burning supergiants and blueward evolution of stars from the ZAMS (caused by chemically-homogeneous evolution). Nonetheless, the MUSE observations have given us a first census of the high-mass population of Leo $\mathrm{P}$, providing confirmed targets for long-exposure spectroscopy to obtain higher $\mathrm{S} / \mathrm{N}$ and better spectral resolution. In particular, we highlight future 
observations of the O-type star (LP 26) to derive its physical properties and a more robust estimate of its mass (cf. predictions from the IGIMF). Moreover, to interpret such observations we will also require synthetic spectra at this low metallicity from the latest model-atmosphere codes.

The MUSE data have also provided a first look at the cool, evolved population of Leo P. Quantitative analysis to determine their physical parameters (in part to calibrate low- $Z$ evolutionary models) and detailed kinematic analysis to investigate the dynamical properties of the cool population will again require ambitious spectroscopic follow-up.

Looking further into the future, the Extremely Large Telescope (ELT) will have the combination of both angular resolution and sensitivity to probe the evolved population of Leo $\mathrm{P}$ in much greater depth, for example with the first-light HARMONI visible and near-IR, integral-field spectrograph (Thatte et al. 2016). Ultimately we also want ultraviolet spectroscopy of the population of massive stars in Leo $\mathrm{P}$, to investigate their wind properties as well as their physical parameters in this important low-metallicity regime. This is unrealistic at present with HST, but would be well within the grasp of the proposed Large Ultraviolet Optical Infrared Surveyor (LUVOIR) concept currently under study (e.g. see Sect. 4.3 from France et al. 2017).

Acknowledgements. Based on observations at the European Southern Observatory Very Large Telescope in programme 094.D-0346. We thank the referee for their constructive and helpful suggestions, and are also grateful to Olivia Jones, Anna McLeod and Rubén Sánchez-Janssen for useful discussions in the course of this work. DRW is supported by a fellowship from the Alfred P. Sloan Foundation, and acknowledges support from the Alexander von Humboldt Foundation. This research made use of Astropy, a community-developed core Python package for Astronomy (Astropy Collaboration 2013), and APLpy, an open-source plotting package for Python (Robitaille \& Bressert 2012).

\section{References}

Aloisi, A., Clementini, G., Tosi, M., et al. 2007, ApJ, 667, L151 Astropy Collaboration, (Robitaille, T. P., et al.) 2013, A\&A, 558, A33 Bacon, R., Accardo, M., Adjali, L., et al. 2010, Proc. SPIE, 7735, 08 Bagnulo, S., Jehin, E., Ledoux, C., et al. 2003, The Messenger, 114, 10 Battinelli, P., \& Demers, S. 2005, A\&A, 434, 657

Bellazzini, M., Oosterloo, T., Fraternali, F., \& Beccari, G. 2013, A\&A, 559, L11 Bernstein-Cooper, E. Z., Cannon, J. M., Elson, E. C., et al. 2014, AJ, 148, 35

Boyer, M. L., Skillman, E. D., van Loon, J. T., Gehrz, R. D., \& Woodward, C. E. 2009, ApJ, 697, 1993

Boyer, M. L., Srinivasan, S., Riebel, D., et al. 2012, ApJ, 748, 40

Boyer, M. L., McQuinn, K. B. W., Barmby, P., et al. 2015, ApJ, 800, 51

Boyer, M. L., McQuinn, K. B. W., Groenewegen, M. A. T., et al. 2017, ApJ, 851, 152

Brott, I., de Mink, S. E., Cantiello, M., et al. 2011, A\&A, 530, A115

Camacho, I., Garcia, M., Herrero, A., \& Simón-Díaz, S. 2016, A\&A, 585, A82

Cannon, J. M., Johnson, M., McQuinn, K. B. W., et al. 2014, ApJ, 787, L1

Castro, N., Crowther, P. A., Evans, C. J., et al. 2018, A\&A, 614, A147

Coe, M. J., Edge, W. R. T., Galache, J. L., \& McBride, V. 2005, MNRAS, 356, 502

Dolphin, A. E. 2000, PASP, 112, 1383

Evans, C. J., Smartt, S. J., Lee, J.-K., et al. 2005, A\&A, 437, 467
Evans, C. J., Taylor, W. D., Hénault-Brunet, V., et al. 2011, A\&A, 530, A108 Finkelstein, S. L., Papovich, C., Ryan, R. E., et al. 2012, ApJ, 758, 93 France, K., Fleming, B., West, G., et al. 2017, Proc. SPIE, 10397, 13 Garcia, M. 2018, MNRAS, 474, L66

Garcia, M., \& Herrero, A. 2013, A\&A, 551, A74

Garcia, M., Herrero, A., Najarro, F., Lennon, D. J., \& Alejandro Urbaneja, M. 2014, ApJ, 788, 64

Georgy, C., Ekström, S., Eggenberger, P., et al. 2013, A\&A, 558, A103 Giovanelli, R., Haynes, M. P., Adams, E. A. K., et al. 2013, AJ, 146, 15 Guha Niyogi, S., Min, M., Meixner, M., et al. 2014, A\&A, 569, A80 Hosek, Jr., M. W., Kudritzki, R.-P., Bresolin, F., et al. 2014, ApJ, 785, 151 Iben, I. J., \& Renzini, A. 1983, ARA\&A, 21, 271

Jeřábková, T., Hasani Zonoozi, A., Kroupa, P., et al. 2018, A\&A, 620, A39 Jones, O. C., Maclay, M. T., Boyer, M. L., et al. 2018, ApJ, 854, 117 Kacharov, N., Rejkuba, M., \& Cioni, M.-R. L. 2012, A\&A, 537, A108 Kamann, S., Husser, T.-O., Wendt, M., et al. 2016, Msngr, 164, 18 Kamann, S., Wisotzki, L., \& Roth, M. 2013, A\&A, 549, A71 Köhler, K., Langer, N., de Koter, A., et al. 2015, A\&A, 573, A71 Lanz, T., \& Hubeny, I. 2003, ApJS, 146, 417

Lanz, T., \& Hubeny, I. 2007, ApJS, 169, 83

Lee, C.-H. 2016, MNRAS, 461, L37

Martins, F., Schaerer, D., \& Hillier, D. J. 2005, A\&A, 436, 1049

Massey, P., Puls, J., Pauldrach, A. W. A., et al. 2005, ApJ, 627, 477

McQuinn, K. B. W., Skillman, E. D., Berg, D., et al. 2013, AJ, 146, 145

McQuinn, K. B. W., Skillman, E. D., Dolphin, A., et al. 2015, ApJ, 812, 158

McQuinn, K. B. W., Boyer, M. L., Mitchell, M. B., et al. 2017, ApJ, 834, 78

Meynet, G., \& Maeder, A. 2002, A\&A, 390, 561

Michałowski, M. J., Murphy, E. J., Hjorth, J., et al. 2010, A\&A, 522, A15

Mokiem, M. R., de Koter, A., Evans, C. J., et al. 2006, A\&A, 456, 1131

Mokiem, M. R., de Koter, A., Evans, C. J., et al. 2007, A\&A, 465, 1003

Morrissey, P., Matuszewski, M., Martin, D. C., et al. 2018, ApJ, 864, 93

Patrick, L. R., Evans, C. J., Davies, B., et al. 2015, ApJ, 803, 14

Pflamm-Altenburg, J., Weidner, C., \& Kroupa, P. 2007, ApJ, 621, 1550

Pustilnik, S. A., Kniazev, A. Y., \& Pramskij, A. G. 2005, A\&A, 443, 91

Rhode, K. L., Salzer, J. J., Haurberg, N. C., et al. 2013, AJ, 145, 149

Robitaille, T., \& Bressert, E. 2012, Astrophysics Source Code Library, [record ascl:1208.017]

Roth, M. M., Sandin, C., Kamann, S., et al. 2018, A\&A, 618, A3

Sacchi, E., Annibali, F., Cignoni, M., et al. 2016, ApJ, 830, 3

Sibbons, L. F., Ryan, S. G., Cioni, M.-R. L., Irwin, M., \& Napiwotzki, R. 2012, A\&A, 540, A135

Skillman, E. D., \& Kennicutt, R. C. 1993, ApJ, 411, 655

Skillman, E. D., Salzer, J. J., Berg, D. A., et al. 2013, AJ, 146, 3

Skrutskie, M. F., Cutri, R. M., Stiening, R., et al. 2006, AJ, 131, 1163

Sloan, G. C., Matsuura, M., Zijlstra, A. A., et al. 2009, Science, 323, 353

Sloan, G. C., Matsuura, M., Lagadec, E., et al. 2012, ApJ, 752, 140

Stark, D. P. 2016, ARA\&A, 54, 761

Szécsi, D., Langer, N., Yoon, S.-C., et al. 2015, A\&A, 581, A15

Tautvaišienè, G., Geisler, D., Wallerstein, G., et al. 2007, AJ, 134, 2318

Thatte, N. A., Clarke, F., Bryson, I., et al. 2016, Proc. SPIE, 9908, 1X

Tramper, F., Sana, H., de Koter, A., \& Kaper, L. 2011, A\&A, 741, L8

Tramper, F., Sana, H., de Koter, A., Kaper, L., \& Ramírez-Agudelo, O. H. 2014, A\&A, 572, A36

Trundle, C., Dufton, P. L., Hunter, I., et al. 2007, A\&A, 471, 625

Valiante, R., Schneider, R., Bianchi, S., \& Andersen, A. C. 2009, MNRAS, 397, 1661

Venn, K. A., Tolstoy, E., Kaufer, A., et al. 2003, AJ, 126, 1326

Walborn, N. R. 1972, AJ, 77, 312

Wegner, W. 2000, MNRAS, 319, 771

Weisz, D. R., \& Boylan-Kolchin, M. 2017, MNRAS, 469, L83

Weisz, D. R., Dolphin, A. E., Skillman, E. D., et al. 2014, ApJ, 789, 147

Williams, B. F., Lang, D., Dalcanton, J. J., et al. 2014, ApJS, 215, 9

Zhukovska, N., \& Henning, T. 2013, A\&A, 555, A99 